\title{
PRÁCTICA DE LA VASECTOMÍA EN UN MUNICIPIO URBANO. CONSIDERACIONES PRÁCTICAS PARA EL SEGUIMIENTO
}

\author{
Marcos Luján, Almudena Vigil', Jorge Turo, Carlos Pascual, Manuel Nevado², Carlos Martín y \\ Vicente Chiva.
}

Unidad de Urología, Laboratorio de Análisis Clínicos' y Unidad de Anatomía Patológica². Hospital Infanta Cristina. Parla. Madrid. España.

Resumen.- OBJETIVO: Establecer un protocolo de seguimiento adecuado basado en el tiempo necesario para conseguir la azoospermia tras la realización de vasectomía, y revisar la frecuencia de complicaciones en nuestro entorno.

MÉTODOS: Análisis retrospectivo de 391 vasectomías. Seguimiento mediante seminograma postvasectomía a los 6 meses de la intervención, y posteriormente con periodicidad trimestral hasta conseguir la azoospermia. Registro de las frecuentaciones en el Servicio de Urgencia de nuestro centro dentro de los 30 días del postoperatorio.

\section{CORRESPONDENCIA}

Marcos Luián Galán

Jefe de Unidad de Urología

Hospital Infanta Cristina

Avda. Nueve de Junio, 2

28981 Parla. Madrid (España).

mlujang@salud.madrid.org

Aceptado para publicar: 21 de julio 2010
RESULTADOS: Durante el seguimiento se han llevado a cabo 567 seminogramas. De las 391 vasectomías realizadas, 275 presentaron al menos una muestra válida para su procesamiento. Al finalizar los 6 meses tras la cirugía un $41,1 \%$ presentaron espermatozoides aislados inmóviles en el seminograma, 9,7\% tras los 9 meses, y 4,7\% al finalizar los 12 meses. El retrasar el primer seminograma de los 6 a los 9 meses tras la intervención supondría un ahorro de 6153,23 Euros anuales en nuestro entorno, con la desventaja de diferir el alta de casi el $60 \%$ de los pacientes intervenidos. La tasa de complicaciones se situó en el 3,1\% /sólo un paciente requirió reintervención e ingreso). No existió diferencia significativa en cuanto al tiempo operatorio en los pacientes con o sin complicación urológica.

CONCLUSIONES: La proporción de pacientes que no alcanzan la azoospermia tras los 6 meses postcirugía es notable. La práctica de la vasectomía parece fiable y relativamente segura en nuestro entorno, con una contenida tasa de complicaciones.

Palabras clave: Vasectomía; Seminograma; Complicaciones.

Summary.- OBJECTIVES: To establish an adequate follow-up protocol based on time to azoospermia achievement after vasectomy. Also, to review the rate of complications in our setting.

METHODS: Retrospective analysis of 391 men who underwent vasectomy. Follow-up was performed by means of semen analysis 6 months after surgery, and then every 3 months until azoospermia was achieved. Data of visits to the emergency unit at our centre were obtained within the first 30 postoperative days. 
RESULTS: During follow-up 567 semen analysis were performed. From 391 vasectomy interventions, 275 had at least one semen sample available and valid for processing. After the first 6 months from surgery, $41.1 \%$ men still presented nonmotile rare sperm in semen analysis, $9.7 \%$ after 9 months, and $4.7 \%$ after 12 months. If semen analysis was postponed from 6 to 9 months after surgery, a total yearly saving of 6, 153.23 Euro would be observed in our setting, but with the drawback of delaying the diagnosis of azoospermia in nearly $60 \%$ of men. Overall complication rate was 3. $1 \%$ lonly one man required hospital admittance and re-intervention). No statistical difference was observed in operative time with regard to the presence or absence of urological complications.

CONCLUSIONS: The percentage of men not achieving azoospermia 6 months after surgery is notorious. Vasectomy practice in our setting seems to be reliable and safe, with a limited rate of complications.

Keywords: Vasectomy. Semen analysis. Complications.

\section{INTRODUCCIÓN}

La vasectomía es un método anticonceptivo seguro y eficaz (1) que se ha popularizado desde hace tiempo en la población española desde su despenalización en Julio de 1983 (2), realizándose aproximadamente unas 70.000 al año en nuestro país (3). Sus ventajas radican en que puede ser realizado con anestesia local y tiene un carácter definitivo.

Aunque el riesgo de embarazo tras la realización de esta cirugía es escaso, es mayoritaria la práctica de realizar un seguimiento tras la misma, durante el que se mantienen medidas anticonceptivas. Dicho seguimiento consiste en la realización de un seminograma que puede repetirse de modo periódico (habitualmente cada 2-3 meses) hasta su negativización (azoospermia). A partir de aquí, el protocolo presenta gran variabilidad según autores. Mientras que algunos proponen la obtención de al menos dos seminogramas con azoospermia, otros estiman suficiente el presentar un solo análisis con dicho resultado (4). Respecto a la necesidad de la negativización completa (azoospermia) del mismo también existe variabilidad: algunos autores propugnan el seguimiento indefinido hasta su negativización mientras que otros estiman suficiente que los espermatozoides encontrados no sean móviles (5). También existe variabilidad en cuanto a la necesidad del análisis anatomopatológico del conducto deferente seccionado, ya que algunos autores cuestionan incluso la necesidad del mismo (4). Por último, aunque la detección de espermatozoides móviles después de la vasectomía se suele catalogar como un fracaso de la misma, algunos autores indican que más del $50 \%$ de estos casos finalmente terminan en éxito sin la necesidad de reintervención (6).

Los objetivos del presente trabajo son establecer un protocolo de seguimiento adecuado basado en el tiempo necesario para conseguir la azoospermia tras la realización de vasectomía, revisar los casos con potencial fracaso de la cirugía (detección de espermatozoides móviles en el seguimiento), así como la frecuencia de complicaciones en nuestro entorno.

\section{MATERIAL Y MÉTODOS}

Estudio retrospectivo. Se han recopilado los registros informáticos de 391 vasectomías consecutivas realizadas en nuestro centro (hospital de nueva creación de 247 camas en un ámbito urbano) desde su apertura el 22 de febrero de 2008 hasta el 31 de diciembre de 2009. La técnica empleada en esta cirugía fue la propuesta por Schmidt (7), y se realizó en quirófano ambulante con anestesia local (mepivacaína $2 \%$ ). Datos como la fecha de la intervención y el tiempo operatorio se incluyeron en la base de datos. Asimismo se han obtenido en soporte electrónico los resultados de los seminogramas postvasectomía realizados hasta el 10 de Marzo de 2010.

El objetivo del seminograma de control postvasectomía es detectar la presencia de espermatozoides en el eyaculado. Se emitió informe que incluyó el volumen total del eyaculado, $\mathrm{pH}$ de la muestra y recuento total de espermatozoides. Este último se realizó mediante dos métodos (8):

- Análisis en fresco: Se homogeneizó la muestra mediante agitación suave y situando una alícuota de 10 $\mu l$ entre portaobjetos y cubreobjetos, se examinaron no menos de 20-30 campos con un microscopio de campo claro a 40x. En caso de detectar la presencia de espermatozoides inmóviles se mencionó en el informe. En el caso de no observar espermatozoides no se consideró un resultado negativo hasta su confirmación mediante el recuento en cámara de $\mathrm{Neu}$ baver.

- Recuento en cámara de Neubauer: Se transfirió la muestra a un tubo con solución salina, y se centrifugó a 1700 rpm (500G) durante 10 minutos. Se eliminó el sobrenadante y se estudió el sedimento (donde se encuentra la concentración de espermatozoides que 
se va a valorar). Se diluyó en solución salina y se realizó una fijación con solución de formaldehído. Finalmente, se cargó en una cámara de Neubauer adaptada a un microscopio de campo claro a un aumento de 40x. Los valores del resultado se expresaron en millones de espermatozoides por $\mathrm{ml}$, tras haber concentrado la muestra 1000 veces, siendo la sensibilidad del método $<0.001$ millones $/ \mathrm{ml}$.

El protocolo de nuestro centro contempla la realización del primer seminograma postvasectomía a los 6 meses de la intervención (aunque en casos aislados se ha llevado a cabo dicho análisis con anterioridad), y posteriormente con periodicidad trimestral de modo indefinido hasta conseguir la azoospermia. Un solo seminograma con azoospermia se considera requisito suficiente para el alta del paciente. Se han excluido del análisis los casos con seguimiento insuficiente (inferior a 7 meses) a no ser que tuvieran al menos un seminograma disponible.

En todos los casos se solicitó estudio anatomopatológico de una sección de cada conducto deferente intervenido, confirmándose en todos los casos que se trataba de conductos deferentes exentos de anomalías.

Adicionalmente se obtuvo otra base de datos con los 153.379 registros de las frecuentaciones registradas en el Servicio de Urgencia desde la apertura del centro hasta el 01/03/2010 con objeto de detectar las atenciones realizadas como consecuencia de la intervención. Se consideraron todas las consultas dentro de los 30 días del postoperatorio y se relacionaron estas con la duración del tiempo operatorio. Las frecuentaciones en el servicio de urgencias fueron catalogadas como:

1) Motivo no urológico (sin relación con la vasectomía),
2) Frecuentación injustificada, o casos en los que el facultativo que lo valora no encuentra patología (dolor leve sin complicación, molestias de la herida, dudas del paciente, etc),

3) Complicación leve, resuelta mediante alta en el Servicio de Urgencia sin requerir intervención quirúrgica ni ingreso hospitalario (infección de herida, hematoma, orquioepididimitis, etc.), y

4) Complicación severa, requiriendo cirugía o ingreso hospitalario.

Los datos de las intervenciones y de las frecuentaciones en el Servicio de Urgencia fueron obtenidas de la aplicación Data WareHouse (Siemens, Oracle Corporation 2005). Los registros informáticos de los seminogramas fueron obtenidos de la aplicación Servolab (DADE-Behring, Siemens Healthcare Diagnostics Inc). El cruce de las bases de datos se realizó con el paquete Microsoft Access 2003 (Microsoft Corp. Chicago IL, USA). El análisis estadístico se llevó a cabo con la aplicación SPSS 13 (SPSS Inc. Chicago IL, USA). Para el análisis de la consecución de la azoospermia a lo largo del seguimiento se empleó el test no paramétrico de Kaplan-Meier. La relación entre la tasa de complicaciones y el tiempo operatorio se estudió mediante el test no paramétrico de Kruskal-Wallis.

\section{RESULTADOS}

Se realizaron un total de 391 vasectomías durante el periodo del estudio. La edad de los pacientes del estudio se muestra en la Tabla l, así como la duración de la intervención.

Durante el seguimiento se han llevado a cabo 567 seminogramas. De las 391 vasectomías realiza-

TABLA I. CARACTERISTICAS DE LOS PACIENTES DEL ESTUDIO (N=391).

\begin{tabular}{|l|c|c|}
\hline & Edad (años) & Tiempo operatorio* (min) \\
\hline Máximo - Mínimo & $28,5-57,7$ & $13-49$ \\
\hline Mediana & 37,1 & 24 \\
\hline Media & 37,5 & 24,6 \\
\hline Error estándar de la media & 0,225 & 0,393 \\
\hline
\end{tabular}

* Incluye tiempo muerto quirúrgico. 
das en el periodo del estudio, 297 disponían de al menos un seminograma postoperatorio, aunque sólo 275 presentaron al menos una muestra válida para su procesamiento. Antes de los 4, 5 y 6 meses de la intervención, 12, 53 y 235 pacientes respectivamente ya disponían de un seminograma. Un total de 28 pacientes no han comparecido para el seguimiento a pesar de haber transcurrido más de 7 meses tras la intervención.

En la Figura 1 se muestra la curva de supervivencia que representa la probabilidad de alcanzar la azoospermia en función del tiempo transcurrido desde la intervención. La probabilidad de persistir espermatozoides inmóviles en el semen estudiado fue del $41,1 \%$ al finalizar los 6 meses del seguimiento, del $9,7 \%$ tras los 9 meses, y del $4,7 \%$ al finalizar los 12 meses.

Adicionalmente se calculó el ahorro potencial de modificar el protocolo vigente en base a los resultados obtenidos. Durante el año 2009 en nuestro centro se realizaron 233 vasectomías. Si hubiéramos aumentado la espera para la realización del primer seminograma de 6 a 9 meses, habríamos ahorrado a 95,8 pacientes (individuos no azoospérmicos a los 6 meses de la intervención) un seminograma $(4,61$ euros / unidad) y una consulta de revisión $(59,62$ euros / unidad). Ello habría supuesto un ahorro de 6153,23 Euros anuales, con la desventaja de diferir el alta de 137,2 pacientes que esperarían 3 meses más para recibirla (individuos azoospérmicos a los 6 meses).

Se observaron únicamente dos casos con espermatozoides móviles en el seguimiento: uno con un primer (y único en el momento de publicar este trabajo) seminograma a los 9 meses de la intervención, donde se observan 111 espermatozoides $130 \%$ móviles) tras concentrar mil veces la muestra. Un segundo caso muestra, tras dos seminogramas con azoospermia, espermatozoides abundantes y móviles en seminograma realizado a los 14 meses de la intervención, con un nuevo seminograma posterior con espermatozoides inmóviles. No se informó de la existencia de embarazos en la serie presentada.

De las 391 vasectomías realizadas, un total de 42 acudieron al Servicio de Urgencia de nuestro centro dentro de los 30 primeros días postcirugía. En la Tabla II se desglosan los motivos. No se observó diferencia significativa en cuanto al tiempo operatorio en los pacientes con o sin complicación urológica $(p=0,320)$. Un paciente fue ingresado y reintervenido a las 24 horas de la intervención por complicación severa (Tabla II), tratándose de un hematoma

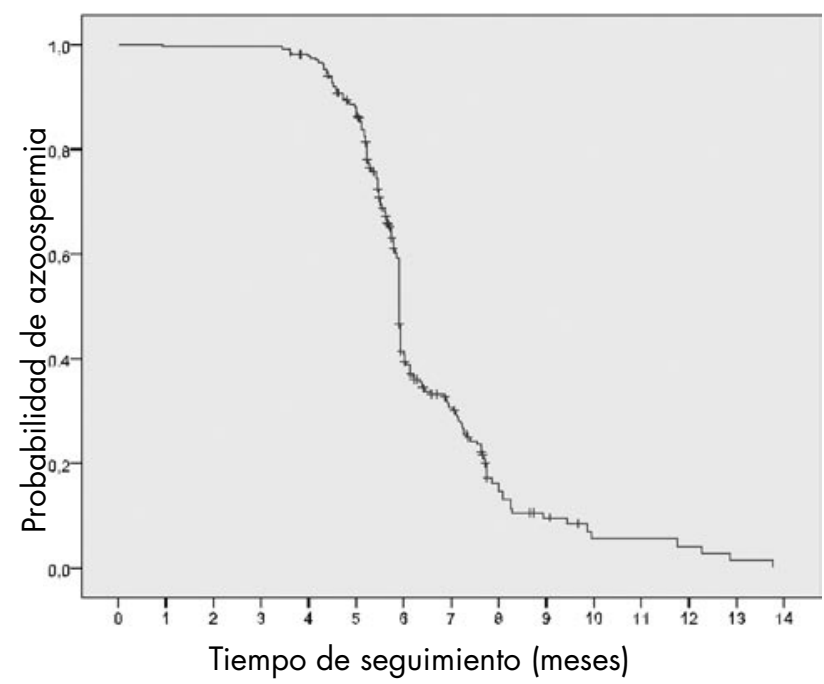

\begin{tabular}{|l|c|c|c|c|c|c|c|c|c|c|c|c|c|c|}
\hline & 1 & 2 & 3 & 4 & 5 & 6 & 7 & 8 & 9 & 10 & 11 & 12 & 13 & 14 \\
\hline $\begin{array}{l}\text { Pacientes al } \\
\text { inicio } \\
\text { del intervalo }\end{array}$ & 275 & 274 & 274 & 274 & 266 & 231 & 77 & 49 & 18 & 10 & 4 & 4 & 3 & 1 \\
\hline $\begin{array}{l}\text { Consiguen } \\
\text { azoospermia }\end{array}$ & 1 & 0 & 0 & 5 & 30 & 110 & 18 & 23 & 6 & 3 & 0 & 1 & 2 & 1 \\
\hline $\begin{array}{l}\text { Persistencia } \\
\text { de espermato- } \\
\text { zoides } \\
\text { al final del } \\
\text { intervalo } \\
\text { (probabilidad) }\end{array}$ & 0,996 & 0,996 & 0,996 & 0,978 & 0,867 & 0,411 & 0,308 & 0,151 & 0,097 & 0,063 & 0,063 & 0,047 & 0,016 & 0,000 \\
\hline
\end{tabular}

FIGURA 1. Curva de supervivencia (Kaplan-Meier) y tabla asociada donde se observa la probabilidad de conseguir la azoospermia en función del tiempo transcurrido desde la intervención. 


\section{TABLA II. FRECUENTACIÓN DEL SERVICIO DE URGENCIA DE LOS PACIENTES DEL ESTUDIO} DENTRO DE LOS 30 DÍAS A PARTIR DE LA FECHA DE INTERVENCIÓN.

\begin{tabular}{|l|c|c|}
\hline & Frecuencia & $\%$ \\
\hline No frecuentación & 349 & 89,3 \\
\hline Complicación leve & 11 & 2,8 \\
\hline Complicación severa & 1 & 0,3 \\
\hline Causa no urológica & 14 & 3,6 \\
\hline Injustificada & 16 & 4,1 \\
\hline Total & 391 & 100,0 \\
\hline
\end{tabular}

postquirúrgico que no se resolvió con medidas conservadoras (apertura escrotal, drenaje y revisión de la hemostasia), sin secuelas posteriores.

\section{DISCUSIÓN}

En este trabajo hemos observado que, transcurridos 6 meses tras la intervención, peristen espermatozoides inmóviles en el semen de $41,1 \%$ de los pacientes, disminuyendo a un $9,7 \%$ a los 9 meses de la intervención.

La proporción de pacientes con azoospermia a los 6 meses postrasectomía (menos del 60\%) es notablemente escasa en nuestro medio, lejos del $80 \%$ a los 3 meses descrito por Griffin y cols (4). En una serie publicada en 2004 con datos de nuestra área sanitaria, Gómez y cols (9) encontraron que se necesitan 200 días (6,6 meses) para que el $80 \%$ de los pacientes alcancen la azoospermia. Basándonos en estos resultados se adoptó en nuestro centro el presente protocolo. Otras series han presentado resultados similares (10).

Una razonable explicación para la persistencia de espermatozoides inmóviles aislados en el semen postvasectomía sería que algunos espermatozoides podrían permanecer en la ampolla deferencial, más tortuosa y formada por múltiples y diminutos compartimentos. Ello haría que el conseguir la azoospermia podría ser impredecible y a menudo muy lento. No obstante, no parece existir mayor riesgo de embarazo si se obtienen espermatozoides aislados inmóviles (como sucede en la casi totalidad de los pacientes con seminograma positivo de nuestra serie), que cuando se documenta la azoospermia (11).

Según los datos actuales, con el protocolo actual el seminograma ha de ser repetido en más del $40 \%$ de los pacientes, lo cual supone un mayor coste de peticiones de laboratorio y de revisiones en consulta. El demorar el primer seminograma postvasectomía a los 9 meses de la intervención haría descender a menos de un 10\% el total de pacientes que requerirían la repetición del mismo, con el consiguiente ahorro para el sistema sanitario (más de 6.000 euros anuales en nuestro ámbito). La otra cara de la moneda es que demoraríamos el alta 3 meses (al pasar de 6 a 9 meses el intervalo hasta la primera revisión tras la vasectomía) a casi el $60 \%$ de los pacientes sometidos a esta cirugía.

En cuanto al hallazgo de espermatozoides móviles en el seminograma lo hemos observado en 2 de 275 pacientes con espermiograma interpretable $(0,7 \%)$. En la literatura este porcentaje oscila entre el 0,3 y el $13 \%$, y no necesariamente conlleva a asumir un fallo de la cirugía, ya que más del $50 \%$ pueden terminar, en futuras revisiones, como un éxito diferido (6). De los dos pacientes de nuestra serie con espermatozoides móviles, uno de ellos los presentó tras dos seminogramas con azoospermia (indicativo de recanalización espontánea) a los 14 meses de la intervención. Un nuevo seminograma posterior constató la persistencia de espermatozoides, esta vez inmóviles. Cabe destacar que el seguimiento de este paciente no se realizó según el protocolo del centro, ya que el propio paciente demandó el seguimiento periódico con seminograma. El otro paciente que presentó espermatozoides móviles lo hizo a los 9 meses de la intervención, estando en la actualidad a la espera de seguimiento.

La tasa de complicaciones relacionadas en nuestra experiencia se situó en el 3,1\%, muy similar a lo observado en la literatura (12). Es incluso superior la tasa de frecuentaciones injustificadas $(4,1 \%)$, debido probablemente a la gran accesibilidad a los recursos públicos de nuestro ámbito. Sólo un caso 
requirió intervención urgente e ingreso hospitalario, resolviéndose satisfactoriamente. No se encontró relación entre el tiempo operatorio y el presentar o no complicación en los días siguientes a la intervención.

La principal limitación del presente estudio es que nuestro protocolo contempla la realización del primer seminograma a los 6 meses tras la intervención. Ello implica que pocos pacientes disponían del mismo antes de dicho periodo, por lo que el comportamiento del seminograma no pudo ser adecuadamente evaluado en los tiempos iniciales del seguimiento de los pacientes. En cualquier caso, a la vista de la elevada tasa de seminogramas que no han alcanzado la azoospermia a los seis meses de la intervención, no parece que realizar seminogramas en un plazo menor esté justificado.

\section{CONCLUSIONES}

La proporción de pacientes que no alcanzan la azoospermia tras los 6 meses postcirugía es notable. El demorar el primer seminograma hasta los 9 meses tras la vasectomía supondría una disminución de los costes, pero obligaría a diferir el alta a casi un $60 \%$ de los pacientes. La práctica de la vasectomía parece fiable y relativamente segura en nuestro entorno, con una contenida tasa de complicaciones.

\section{AGRADECIMIENTOS}

A María Teresa Sánchez Alvarez, del Departamento de Control de Gestión del Hospital Infanta Cristina (Parla, Madrid) por su ayuda en la obtención de los registros de las frecuentaciones del Servicio de Urgencia.

A Juan Manuel Fernández, del Laboratorio de Análisis Clínicos del Hospital Infanta Cristina (Parla, Madrid), por su ayuda en el suministro de los datos de los seminogramas postvasectomía.

A Juan Torres Macho, coordinador del Servicio de Urgencia del Hospital Infanta Cristina (Parla, Madrid).

\section{BIBLIOGRAFÍA y LECTURAS RECOMENDADAS ( ${ }^{*}$ lectura de interés $y^{* *}$ lectura fundamental)}

**1. Schwingl PJ, Guess HA. Safety and effectiveness of vasectomy. Fertil Steril 2000; 73: 923-936.

2. El País 01/05/1985, último acceso 15/03/2010: http://www.elpais.com/articulo/madrid/MADRID/centros/salud/incorporan/vasectomia/ programas/anticonceptivos/elpepiautmad/ 19850501elpmad_7/Tes/.

3. Europa Press 16/06/2008, último acceso 15/03/2010: http://www.europapress.es/salud/noticia-70000-varones-aplican-cada-ano-espana-vasectomia-20080616215958.html

**4. Griffin T, Tooher R, Nowakowski K, Lloyd M, Maddern G. How little is enough? The evidence for post-vasectomy testing. J Urol 2005; 174: 29 36.

*5. De Knijff DW, Vrijhof HJ, Arends J, Janknegt RA. Persistence or reappearance of nonmotile sperm after vasectomy: does it have clinical consequences? Fertil Steril 1997; 67: 332-335.

*6. Labrecque M, St-Hilaire K, Turcot L. Delayed vasectomy success in men with a first postvasectomy semen analysis showing motile sperm. Fertil Steril 2005; 83(5): 1435-1441.

7. Schmidt SS. Vasectomy by section, luminal fulguration and fascial interposition: results from 6248 cases. Br J Urol 1995; 76: 373-375.

8. Aulesa C y Castilla JA. Recomendaciones en el estudio del semen posvasectomía y vasovasostomía. Química Clínica 2007; 26(2): 73-76.

9. Gómez JM, Romero I, Blanco C, Pastor J, Moreno A, Santos D, y cols. La historia natural del aclaramiento de espermatozoides en el semen tras la vasectomía. Actas Urol Esp 2004; 28 (4): 286289.

10. Smith AG, Crooks J, Singh NP, Scott R, Lloyd SN. Is the timing of post-vasectomy seminal analysis important? Br J Urol 1998; 81: 458-460.

**11. Haldar N, Cranston D, Turner E, MacKenzie I, Guillebaud J. How reliable is a vasectomy? Longterm follow-up of vasectomised men. Lancet 2000; 356: 43-44.

12. Romero P, Merenciano FJ, Rafie W, Amat M, Martínez MC. La vasectomía: estudio de 300 intervenciones. Revisión de la literatura nacional y de sus complicaciones. Actas Urol Esp 2004; 28(3): $175-214$ 